Pacific Journal of Mathematic 


\section{A REPRESENTATION THEOREM FOR CONVOLUTION TRANSFORM WITH DETERMINING FUNCTION IN $L^{p}$.}

\section{Chaim Danon and Dany Leviatan}

Let $G(t)$ be a kernel in Class II. Necessary conditions in order that a function $f(x)$ be the convolution transform of $\phi(t) \in L^{p}(-\infty, \infty)$ were obtained by the second author. Also it was conjectured that the conditions are in fact sufficient. The conjecture is indeed true and we prove it here.

Following the notation of [3] (see [3] §2) we have

THEOREM. Necessary and sufficient conditions in order that $f(x)$ possess the representation

$$
f(x)=\int_{-\infty}^{\infty} G(x-t) \phi(t) d t, \quad \gamma<x<\infty
$$

where $\phi(t) \in L^{p}(-\infty, \infty)(1<p<\infty)$ are that $f(x) \in C^{\infty}(\gamma, \infty)$ and that

$$
\sup _{\gamma<x<\infty} \sum_{n=0}^{\infty} \frac{1}{a_{n+1}}\left|f_{n}\left(x-\lambda_{n}\right)\right|^{p} \equiv H<\infty
$$

Furthermore,

$$
\int_{-\infty}^{\infty}|\phi(t)|^{p} d t=H
$$

Necessity follows from [3], Theorem 2 for $M(u)=|u|^{p}$. The equality (2) is established in the proofs of Theorems 2, 3 in [3]. For the sufficiency we shall need the following lemmas,

LEMMA 1. For every $\tau>0$,

$$
\sum_{n=0}^{\infty} \frac{1}{a_{n+1}} H_{n+1}\left(-\tau+\lambda_{n+1}\right)=1
$$

Proof. By [3] (3.10),

$$
\sup _{-\infty<\theta<\infty} \sum_{n=0}^{\infty} \frac{1}{a_{n+1}} G_{n}\left(\theta-\lambda_{n}\right) \leqq 1
$$


Thus, $G(x)$ satisfies [3], (3.3) that is for $-\infty<x<u<\infty$,

$$
G(x)=\sum_{k=0}^{\infty} \frac{1}{a_{k+1}} G_{k}\left(u-\lambda_{k}\right) H_{k+1}\left(x-u+\lambda_{k+1}\right) .
$$

Put $u=x+\tau(\tau>0)$ we have,

$$
\begin{aligned}
1 & =\int_{-\infty}^{\infty} \sum_{k=0}^{\infty} \frac{1}{a_{k+1}} G_{k}\left(x+\tau-\lambda_{k}\right) H_{k+1}\left(-\tau+\lambda_{k+1}\right) d x \\
& =\sum_{k=0}^{\infty} \frac{1}{a_{k+1}} H_{k+1}\left(-\tau+\lambda_{k+1}\right) \int_{-\infty}^{\infty} G_{k}\left(x+\tau-\lambda_{k}\right) d x \\
& =\sum_{k=0}^{\infty} \frac{1}{a_{k+1}} H_{k+1}\left(-\tau+\lambda_{k+1}\right) .
\end{aligned}
$$

LEMMA 2. If $f(x) \in C^{\infty}(\gamma, \infty)$ and satisfies (1), then for every $\gamma<$ $x<u<\infty$,

$$
f(x)=\sum_{k=0}^{\infty} \frac{1}{a_{k+1}} f_{k}\left(u-\lambda_{k}\right) H_{k+1}\left(x-u+\lambda_{k+1}\right) .
$$

Proof. We first show that the series converges.

By Hölder's inequality we have

$$
\begin{aligned}
I & \equiv \sum_{k=N}^{\infty} \frac{1}{a_{k+1}}\left|f_{k}\left(u-\lambda_{k}\right)\right| H_{k+1}\left(x-u+\lambda_{k+1}\right) \\
& \leqq\left(\sum_{k=N}^{\infty} \frac{1}{a_{k+1}}\left|f_{k}\left(u-\lambda_{k}\right)\right|^{p}\right)^{1 / p}\left(\sum_{k=N}^{\infty} \frac{1}{a_{k+1}} H_{k+1}^{q}\left(x-u+\lambda_{k+1}\right)\right)^{1 / q} .
\end{aligned}
$$

For $N$ sufficiently large, the $H_{k}$ 's are uniformly bounded (say by $M$ ) (see [3] p. 444) and since $q>1$,

$$
\begin{aligned}
I & \leqq H^{1 / p} M^{1 / p}\left(\sum_{k=N}^{\infty} \frac{1}{a_{k+1}} H_{k+1}\left(x-u+\lambda_{k+1}\right)\right)^{1 / q} \\
& \leqq(H M)^{1 / p} \quad \text { by Lemma } 1 .
\end{aligned}
$$

Now, by the Lemma in [3] p. 442 for every $\gamma<x<u<\infty$,

$$
f(x)=\sum_{k=0}^{n} \frac{1}{a_{k^{\prime}+1}} f_{k}\left(u-\lambda_{k}\right) H_{k+1}\left(x-u+\lambda_{k+1}\right)+R_{n}(x, u)
$$

where

$$
R_{n}(x, u)=\int_{x}^{u} f_{n+1}\left(t-\lambda_{n+1}\right) H_{n+1}\left(x-t+\lambda_{n+1}\right) d t
$$


Since the series converges, so does $R_{n}(x, u)$. In order to show that $R_{n}(x, u) \rightarrow 0(n \rightarrow \infty)$, it suffices to find some subsequence $\left\{n_{j}\right\}$ such that $R_{n} \rightarrow 0(j \rightarrow \infty)$. To this end notice that (1) implies

$$
\sum_{n=0}^{\infty} \frac{1}{a_{n+1}} \int_{x}^{u}\left|f_{n}\left(t-\lambda_{n}\right)\right|^{p} d t<\infty
$$

and since $\sum_{n=0}^{\infty}\left(1 / a_{n+1}\right)=\infty$, there exists a subsequence $\left\{n_{j}\right\}$ such that

$$
\int_{x}^{u}\left|f_{n_{j}}\left(t-\lambda_{n_{t}}\right)\right|^{p} d t \rightarrow 0, \quad j \rightarrow \infty
$$

By Hölder's inequality,

$$
\int_{x}^{u}\left|f_{n_{j}}\left(t-\lambda_{n_{j}}\right)\right| d t \rightarrow 0, \quad j \rightarrow \infty
$$

and since $H_{n}(t)$ are uniformly bounded, $R_{n}(x, u) \rightarrow 0, j \rightarrow \infty$.

LEMMA 3. The series

$$
\sum_{n=0}^{\infty} \frac{1}{a_{n+1}} G\left(\theta+\lambda_{n+1}\right)
$$

is uniformly bounded in $-\infty<\theta<\infty$.

Proof. It is known that $G(x)$ is bell shaped and non negative. (See [1], p. 126). Let $G\left(x_{0}\right)=\max G(x)$. Then $G$ increases to the left of $x_{0}$ and decreases beyond $x_{0}$. Hence

$$
\sum_{\theta+\lambda_{n+1}>x_{0}} \frac{1}{a_{n+1}} G\left(\theta+\lambda_{n+1}\right) \leqq \int_{x_{0}}^{\infty} G(t) d t+\frac{1}{a_{1}} G\left(x_{0}\right) \leqq 1+\frac{1}{a_{1}} G\left(x_{0}\right)
$$

and

$$
\begin{aligned}
0 & \leqq \sum_{\theta+\lambda_{n+1} \leqq x_{0}} \frac{1}{a_{n+1}} G\left(\theta+\lambda_{n+1}\right)-\int_{-\infty}^{x_{0}} G(t) d t \\
& \leqq \sum_{\theta+\lambda_{n+1} \leqq x_{0}} \frac{1}{a_{n+1}}\left[G\left(\theta+\lambda_{n+1}\right)-G\left(\theta+\lambda_{n}\right)\right] \\
& =\sum \frac{1}{a_{n+1}}\left(\lambda_{n+1}-\lambda_{n}\right) G^{\prime}\left(\theta+\mu_{n}\right) \quad\left(\lambda_{n}<\mu_{n}<\lambda_{n+1}\right) \\
& \leqq \sup _{-\infty<t<\infty}\left|G^{\prime}(t)\right| \sum_{n=0}^{\infty} \frac{1}{a_{n+1}^{2}} .
\end{aligned}
$$


REMARKs. (a) The same proof applied to $G_{k}(t)(k=1,2, \cdots)$ gives the same for the series $\sum_{n=0}^{\infty}\left(1 / a_{n+1}\right) G_{k}\left(\theta+\lambda_{n+1}\right)$. (b) Some slight modifications in this analysis yield that:

$$
\lim _{\tau \rightarrow \infty} \sum_{n=0}^{\infty} \frac{1}{a_{n+1}} G\left(-\tau+\lambda_{n+1}\right)=1
$$

LEMMA 4. If $f(x) \in C^{\infty}(\gamma, \infty)$ and satisfies (1), then for $\gamma<x<\infty$,

$$
f(x)=\lim _{u \rightarrow \infty} \sum_{k=0}^{\infty} \frac{1}{a_{k+1}} f_{k}\left(u-\lambda_{k}\right) G\left(x-u+\lambda_{k+1}\right) .
$$

Proof. By Lemma 2 and Hölder's inequality,

$$
\begin{aligned}
\mid f(x) & -\sum_{k=0}^{\infty} \frac{1}{a_{k+1}} f_{k}\left(u-\lambda_{k}\right) G\left(x-u+\lambda_{k+1}\right) \mid \\
\leqq & \sum_{k=0}^{\infty} \frac{1}{a_{k+1}}\left|f_{k}\left(u-\lambda_{k}\right)\right|\left|H_{k+1}\left(x-u+\lambda_{k+1}\right)-G\left(x-u+\lambda_{k+1}\right)\right| \\
\leqq & \left(\sum_{k=0}^{\infty} \frac{1}{a_{k+1}}\left|f_{k}\left(u-\lambda_{k}\right)\right|^{p}\left|H_{k+1}\left(x-u+\lambda_{k+1}\right)-G\left(x-u+\lambda_{k+1}\right)\right|\right)^{1 / p} \\
& \times\left(\sum_{k=0}^{\infty} \frac{1}{a_{k+1}}\left|H_{k+1}\left(x-u+\lambda_{k+1}\right)-G\left(x-u+\lambda_{k+1}\right)\right|\right)^{1 / q} .
\end{aligned}
$$

$H_{n}(t) \rightarrow G(t)$ uniformly in $(-\infty, \infty)$ as $n \rightarrow \infty$ and for each fixed $n$, $H_{n}(t) \rightarrow 0, G(t) \rightarrow 0$ as $t \rightarrow-\infty$. Hence, it follows by (1) that for $\gamma<x<\infty$, the first series converges to zero as $u \rightarrow \infty$, while the second series is bounded uniformly in $x, u$ by Lemmas 1 and 3 .

We now proceed with the proof of the theorem.

Proof of sufficiency. Define the functions $\alpha_{u}(t), u>\gamma$ by

$$
\alpha_{u}(t) \equiv\left\{\begin{array}{cc}
0, & t=0 . \\
\sum_{u>\lambda_{n+1} \geqq u-t} \frac{1}{a_{n+1}} f_{n}\left(u-\lambda_{n}\right), & t>0 . \\
\sum_{u \leqq \lambda_{n+1}<u-t} \frac{1}{a_{n+1}} f_{n}\left(u-\lambda_{n}\right), & t<0 .
\end{array}\right.
$$

For fixed $t, 0<t<\infty$,

$$
\underset{[0, t]}{\operatorname{Var}}\left\{\alpha_{u}(t)\right\} \leqq \sum_{u>\lambda_{n+1} \geqq u-t} \frac{1}{a_{n+1}}\left|f_{n}\left(u-\lambda_{n}\right)\right|
$$




$$
\begin{aligned}
& \leqq\left(\sum \frac{1}{a_{n+1}}\left|f_{n}\left(u-\lambda_{n}\right)\right|^{p}\right)^{1 / p}\left(\sum_{u>\lambda_{n+1} \geqq u-1} \frac{1}{a_{n+1}}\right)^{1 / q} \\
& \leqq H^{1 / p}\left(\lambda_{k+1}-\lambda_{r}\right)^{1 / q}
\end{aligned}
$$

where $\lambda_{k+2}>u>\lambda_{k+1}$ and $\lambda_{r+1} \geqq u-t>\lambda_{r}$. Hence

$$
\underset{[0, t]}{\operatorname{Var}}\left\{\alpha_{u}(t)\right\} \leqq H^{1 / p}\left(t+\frac{1}{a_{r+1}}\right)^{1 / q}
$$

Similar analysis is done for $-\infty<t<0$. Hence $\alpha_{u}(t)$ are of variations uniformly bounded in every finite interval in $(-\infty, \infty)$. By Helly (see [4] p. 29) it can be shown that there is a sequence $u_{i} \uparrow \infty$ and a function $\alpha(t)$ of bounded variation in every finite interval such that $\alpha_{u_{j}}(t) \rightarrow \alpha(t)$ for $-\infty<t<\infty$. Lemma 4 implies

$$
f(x)=\lim _{u_{j} \rightarrow \infty} \int_{-\infty}^{\infty} G(x-t) d \alpha_{u_{l}}(t), \quad y<x<\infty .
$$

For every $t,\left|\alpha_{u}(t)\right| \leqq \operatorname{Var}\left\{\alpha_{u}(t)\right\} \leqq H^{1 / p}\left(|t|+1 / a_{1}\right)^{1 / q}$ and since $t G(t) \rightarrow 0$ as $|t| \rightarrow \infty$, integration by parts yields

$$
f(x)=\lim _{u_{j} \rightarrow \infty} \int_{-\infty}^{\infty} G^{\prime}(x-t) \alpha_{u_{j}}(t) d t, \quad \gamma<x<\infty .
$$

Now $G^{\prime}(t)\left(|t|+1 / a_{1}\right)^{1 / q}$ is integrable so that by Lebesgue's dominated convergence theorem

$$
f(x)=\int_{-\infty}^{\infty} G^{\prime}(x-t) \alpha(t) d t=\int_{-\infty}^{\infty} G(x-t) d \alpha(t) .
$$

We conclude the proof as in [3] p. 448 and obtain that $\alpha(t)$ is the indefinite integral of a function $\phi(v) \in L^{p}(-\infty, \infty)$.

REMARKS. (c) An analogous representation theorem involving integral conditions can be found in [1] p. 153. Notice that we allow here representation in a half line, however, by Hölder's inequality, the integral $\int_{-\infty}^{\infty} G(x-t) \phi(t) d t$ converges for every $-\infty<x<\infty$. Therefore condition (1) (which is required on a half line) defines a convolution transform on $(-\infty, \infty)$.

(d) $H$ is independent of $\gamma$ and is given by

$$
H=\lim _{x \rightarrow \infty} \sum_{n=0}^{\infty} \frac{1}{a_{n+1}}\left|f_{n}\left(x-\lambda_{n}\right)\right|^{p}
$$


(e) For $a_{n} \equiv n$, the convolution transform reduces to the Laplace transform and our therorem yields [2], Theorem 1 for $\phi(t) \in L^{p}(0, \infty)$.

(f) $f(x) \equiv 1$ cannot be the convolution transform of a function $\phi(t) \in L^{p}(-\infty, \infty)$ since

$$
\sum \frac{1}{a_{n+1}}\left|f_{n}\left(x-\lambda_{n}\right)\right|^{p}=\Sigma \frac{1}{a_{n+1}}=\infty .
$$

(g) Denote by $X$ the set of real valued functions which belong to $C^{\infty}(-\infty, \infty)$ and satisfy (1). $\|f\|=H^{1 / p}$ is a norm on $X$. Our representation theorem says that $f \in X$ if and only if $f=G * \phi$ where $\phi \in L^{p}(-\infty, \infty)$. Furthermore, $\|f\|=\|\phi\|$. Equivalently, $X$ and $L^{p}(-\infty, \infty)$ are isomorphic isometric.

Acknowledgement. We are grateful to Dr. Zeev Schuss for most valuable discussions during the preparation of this work.

\section{REFERENCES}

1. I. I. Hirchman and D. V. Widder, The Convolution Transform, Princeton Univ. Press, 1955.

2. D. Leviatan, On the representation of functions as Laplace integrals, J. London Math. Soc., 44 (1969), 88-92.

3. - A new approach to representation theory for convolution transforms, Pacific J. Math., 35 (1970), 441-449.

4. D. V. Widder, The Laplace Transform, Princeton Univ. Press, 1946.

Received June 3, 1974.

TEL-AVIV UNIVERsity 



\section{Pacific Journal of Mathematics}

\section{Vol. 62, No. $1 \quad$ January, 1976}

Mieczyslaw Altman, Contractor directions, directional contractors and

directional contractions for solving equations . .................. 1

Michael Peter Anderson, Subgroups of finite index in profinite groups .........

Zvi Arad, Abelian and nilpotent subgroups of maximal order of groups of odd order

John David Baildon and Ruth Silverman, On starshaped sets and Helly-type theorems ..........................................

John W. Baker and R. C. Lacher, Some mappings which do not admit an

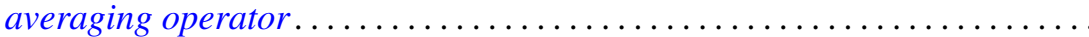

Joseph Barback, Composite numbers and prime regressive isols . . . . . . . . . .

David M. Boyd, Composition operators on $H^{p}(A) \ldots \ldots \ldots \ldots \ldots \ldots \ldots$

Maurice Chacron, Co-radical extension of PI rings . . . . . . . . . . . . .

Fred D. Crary, Some new engulfing theorems . . . . . . . . . . . . . . .

Victor Dannon and Dany Leviatan, A representation theorem for convolution transform with determining function in $L^{p} \ldots \ldots \ldots \ldots \ldots \ldots \ldots \ldots \ldots \ldots \ldots \ldots \ldots \ldots$

Mahlon M. Day, Lumpy subsets in left-amenable locally compact

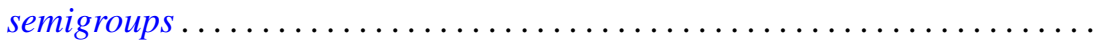

Michael A. Gauger, Some remarks on the center of the universal enveloping algebra of a classical simple Lie algebra . .

David K. Haley, Equational compactness and compact topologies in rings

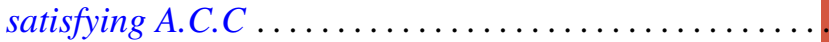

Raymond Heitmann, Generating ideals in Prüfer domains .

Gerald Norman Hile, Entire solutions of linear elliptic equations with

Laplacian principal part. .

Richard Oscar Hill, Moore-Postnikov towers for fibrations in which $\pi_{1}$ (fiber) is non-abelian

John Rast Hubbard, Approximation of compact homogeneous maps . .

Russell L. Merris, Relations among generalized matrix functions . .

V. S. Ramamurthi and Edgar Andrews Rutter, On cotorsion radicals ...

Ralph Tyrrell Rockafellar and Roger Jean-Baptiste Robert Wets, Stochastic

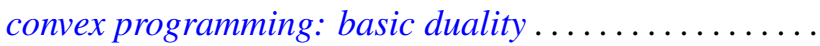

Alban J. Roques, Local evolution systems in general Banach spaces ..

I. Bert Russak, An indirect sufficiency proof for problems with bounded state variables.

Richard Alexander Sanerib, Jr., Ultrafilters and the basis property. .

H. A. Seid, The decomposition of multiplication operators on $L_{p}$-spaces . .

Franklin D. Tall, The density topology .................. 